\title{
The Role of Sensory Modulation Deficits and Behavioral Symptoms in a Diagnosis for Early Childhood
}

\author{
Ruth Pérez-Robles · Eduardo Doval • \\ Ma Claustre Jané • Pedro Caldeira da Silva • \\ Ana Luisa Papoila · Daniel Virella
}

Published online: 2 October 2012

(C) Springer Science+Business Media, LLC 2012

\begin{abstract}
To contribute to the validation of the sensory and behavioral criteria for Regulation Disorders of Sensory Processing (RDSP) (DC:0-3R, 2005), this study examined a sample of toddlers in a clinical setting to analyze: (1) the severity of sensory modulation deficits and the behavioral symptoms of RDSP; (2) the associations between sensory and behavioral symptoms; and (3) the specific role of sensory modulation deficits in an RDSP diagnosis. Based on clinical observations, 78 toddlers were classified into two groups: toddlers with RDSP $(\mathrm{N}=18)$ and those with "other diagnoses in Axis I/II of the DC:0-3R" (OD3R; $\mathrm{N}=60$ ). The parents completed the Infant Toddler Sensory Profile and the Achenbach Checklist. The results revealed that the RDSP group had more severe sensory modulation deficits and specific behavioral symptoms; stronger, although not significant, associations between most sensory and behavioral symptoms; and a significant sensory modulation deficit effect. These findings support the validity of RDSP.
\end{abstract}

R. Pérez-Robles $(\bowtie) \cdot$ M. C. Jané

Departament of Clinical and Health Psychology, Universitat Autònoma de Barcelona, 08193 Bellaterra, Barcelona, Spain e-mail: ruthprobles@gmail.com

R. Pérez-Robles · P. Caldeira da Silva

Infancy and Early Childhood Unit, Department of Child and Adolescent Psychiatry, CHLC-Hospital Dona Estefânia,

Lisbon, Portugal

E. Doval

Departament of Psychobiology and Methodology of Health Sciences, Universitat Autònoma de Barcelona, Barcelona, Spain

A. L. Papoila · D. Virella

Research Center of CHLC-Hospital Dona Estefânia, Lisbon, Portugal
Keywords Sensory modulation dysfunction - Regulation Disorders of Sensory Processing - DC:0-3R - Infant toddler sensory profile $\cdot$ CBCL 1 1/2-5

\section{Introduction}

Regulation Disorders of Sensory Processing (RDSP) is a primary diagnosis in the Diagnostic Classification of Mental Health and Development Disorders of Infancy and Early Childhood revised version: DC:0-3R [1]. This classification and the previous version DC:0-3 [2] emerged as a complement to the Diagnostic and Statistical Manual of Mental Disorders (DSM) in early childhood diagnosis. Their focus on the parent-child relationship taps into important aspects of young children's functioning that are not captured in the DSM-IV-TR [3]. In addition, "this classification emphasized RDSP more than the DSM does" [4].

In DC:0-3R, RDSP is characterized by: "difficulties in regulating emotions and behaviors, as well as motor abilities, in response to sensory stimulation that lead to impairment in development and functioning. These behaviors occur in multiple relationships and contexts" [1]. RDSP can be classified into three types and two subtypes based on the child's sensory threshold (high or low) and his or her self-regulation response type (active or passive). DC:0-3R describes the specific behavioral, sensory and motor patterns that characterize each of these types and subtypes:

Type I "Hypersensitive" describes children with a low sensory threshold. Depending on their self-regulatory strategies, Type I may be classified into the following subtypes: 
Subtype A-"Fearful/cautious": The sensory reactivity patterns of these children are characterized by over-reactivity to sensory stimuli; they appear frightened by nearby sounds, movements, or visual stimuli. The motor patterns are characterized by limited sensory-motor play and less exploration than expected according to age. The behavioral patterns are characterized by excessive cautiousness, inhibition and fearfulness.

Subtype B-"Negative-defiant": The sensory reactivity patterns are identical to those of subtype A. The motor patterns of these children are characterized by avoiding behaviors or being slow to engage in new experiences. Behavioral patterns include: negativistic behavior; controlling behaviors; defiance; and, in general, aggression only when provoked.

Type II "Hyposensitive/Under-responsive" has a high sensory threshold. The sensory reactivity patterns are characterized by under-reactivity to sounds, movement, smell, taste, touch and/or propioception. The motor patterns include: limited exploration; restricted play repertoire; lethargy; poor motor planning and/or searching for specific sensory input. The behavioral patterns are characterized by an apparent lack of interest in exploring properties of objects, playing challenging games, or engaging in social interactions; apathetic appearance; fatigability and/or inattentiveness.

Type III "Sensory-Stimulation Seeking/Impulsive" also has a high sensory threshold but these children use active self-regulation strategies. The sensory reactivity patterns are characterized by the child's craving for high-intensity sensory stimuli. The motor patterns are characterized by a high need for motor discharge, diffuse impulsivity and/or accident proneness without clumsiness. The behavioral patterns can include seeking constant contact with people and objects, recklessness, and disorganized behavior as a consequence of sensory stimulation $[1,5,6]$.

Longitudinal studies [7,8] have shown that $95 \%$ of infants who exhibit moderate symptoms of Regulatory Disorders (RD, as defined in DC:0-3, 1994) within their first year of life were later diagnosed at the age of 3 with delays in motor, language, and cognitive development or relational disorders. Although few epidemiological studies have been conducted in Europe, a study conducted in the Danish population found that $7 \%$ of 1.5 -year-olds have RDSP symptoms [9].

Although there is a broad consensus concerning the clinical usefulness of this diagnosis, data supporting RDSP as a distinct and valid disorder are scant [1]. The RDSP diagnosis originated from clinical observations of toddlers, and its diagnostic criteria were developed via a clinical consensus. This approach is different from that of other diagnoses listed in the DC: 0-3R that have adopted criteria from adult diagnoses. The lack of empirical data led the task force members to conclude that there was insufficient evidence to support the inclusion of detailed symptom criteria for each of the subtypes. Instead, descriptive information was provided with the hope that future research will lead to more specific criteria [1].

The DC:0-3 framework highlights that if both behavioral and sensory symptoms were not present in the child, "the clinician should consider alternative classifications in order to distinguish this diagnosis from others that might have phenotypic similarities in Axis I or II" [1, 2]. For example, fearfulness, as described in Subtype A: Hypersensitive Regulation Disorder may reflect an Anxiety Disorder (Axis I: Primary Diagnosis); negative and "willful" behavior may arise from coercive parenting (Axis II: Relationship Classification)" [1]. Symptoms like "withdrawn or apathetic behavior"; "avoiding behavior"; "freezing"; and "oppositionist behavior and defiance" in the child can also be interpreted by the clinician as characteristics of a deregulated parent-child relationship in Axis II. Consequently, related to the discriminative validity of RDSP, it was very important in the present study to analyze the similarities with other diagnoses of Axis I and Axis II of DC:0-3R.

Although the work group that elaborated the criteria for RDSP in DC: 0-3R also sought input from occupational therapists [10], some overlaps in the classifications of this diagnosis in the field of occupational therapy (OT) and child psychiatry still remain. For example, in OT, Sensory Modulation Disorder (SMD) is a very similar diagnosis to RDSP. Nevertheless, if motor problems are also present, an occupational therapist will typically make a diagnosis of Sensory Based Motor Disorder (SBMD) [10]. The necessity to build bridges between these two areas was mentioned by Miller et al. [10]. This terminology employed in OT was also recently incorporated into the Diagnostic Manual of the Interdisciplinary Council for Developmental and Learning Disorders (ICDL-DMIC) [11].

Despite their clinical importance, the lack of studies validating RDSP was the reason for its exclusion from the Diagnostic and Statistical Manual of Mental Disorders (DSM-IV-TR) [3] and The International Statistical Classification of Diseases and Related Health Problems (ICD10) [12]. The need to study the validity of this diagnosis has been addressed by various authors [4], who noted that the DC:0-3 diagnostic framework diagnoses were not developed using the standard procedures for assessing reliability and validity. These standard procedures use a sample that includes "cases" with diverse problems as well as "non-cases" to validate diagnoses with data not used in the diagnostic process. 
Some of the most frequently-asked questions which have arisen in several studies related to the validity of RDSP diagnoses are presented below:

The first question involves the ability of RDSP sensory and behavioral symptoms (like oppositional behavior; inattention; withdrawn behavior; avoiding behavior; feeding difficulties...) to distinguish between this and other psychiatric diagnosis in early childhood in Axis I (like Posttraumatic Stress Disorder, Affect Disorder, Sleep Disorder and Feeding Disorder) or a diagnosis on Axis II of DC:0-3R. Relatedly, several studies have noted the phenotypic similarities between RDSP and other diagnoses in toddlers [7, 10, 13-16] such as anxiety [17] or other emotional problems [18], Autism Spectrum Disorders (ASD) [13] and other "relationship-based diagnoses" [19]. This similarity has led researchers to question whether RDSP symptoms can help to distinguish RDSP from the other psychiatric disorders that affect children [20, 21].

Although some studies have characterized RDSP symptoms $[8,22]$ and defined its qualities with regard to other diagnoses [13], they used small sample sizes. Furthermore, although some of these studies have analyzed both the sensory and behavioral symptoms of RDSP [23], most have focused exclusively on one or the other. This limitation was partially because there is a similar diagnosis to RDSP known as SMD in OT which is diagnosed primarily using sensory criteria.

This overlap has led to a second question that is also related to the validation of the RDSP diagnosis: Are some of the sensory symptoms of RDSP the same as certain behavioral symptoms when analyzed from different perspectives (i.e., child psychiatry vs. OT)? This question suggests that there is a need to analyze the relationship between these symptoms [17, 24-26].

Relatedly, some studies which examined the co-occurrence of sensory regulation dysfunction and preschool psychiatric disorders with standard measures (and measures in which experts had been used to deliberately separate behavioral and sensory symptoms) have concluded that (depending upon the impairment criteria used) $33-63 \%$ of children meeting the criteria for sensory deregulation also had a psychiatric disorder; $37-67 \%$ only had a sensory deregulation disorder, which indicates that sensory regulation dysfunction exists independently of psychiatric disorder [25].

Nevertheless, some behavioral symptoms are associated with certain sensory patterns to some extent. In this sense, anxiety is often associated with sensory hyper-reactivity $[27,28]$ and inhibition to novel stimuli [29] in both clinical and non-clinical populations. Other studies have shown that affective symptoms tend to persist in children after 2 or 3 years of age, with a higher incidence when accompanied by sensory modulation deficits [18]. Therefore, studies should analyze whether behavioral and sensory symptoms reflect different concepts as well as whether the association between these symptoms is higher in RDSP diagnoses compared with other psychiatric disorders of the DC: 0-3R.

The third question required to validate the RDSP diagnosis refers to the need to study the role of sensory and behavioral symptoms $[21,30]$. Specifically, it is necessary to analyze how the sensory symptoms contribute to the RDSP diagnosis. Determining the important role played by sensory symptoms (which differ from the behavioral symptoms) in RDSP would corroborate their relevance with regard to the diagnostic criteria.

Another question related to the validity of RDSP is its stability over time. Namely, some symptoms of RDSP in preschoolers can be similar to other DSM diagnoses [20,31]. Although not discussed in the present paper, it is important to consider this question in further follow-up studies.

Based on the aforementioned questions, this paper has the following goals:

1. To compare the severity of the behavioral and sensory symptoms present in children diagnosed with RDSP using the DC:0-3R with a sample of children who have "other diagnoses in Axis I/II of the DC:0-3R" (OD3R). The initial hypothesis is that the severity of the symptoms included in the diagnostic criteria of RDSP (inhibition or defiance behaviors, inattention and/or lack of responsivity depending on the RDSP type) will be higher in children with this diagnosis than in children with OD3R. Both of these sensory and behavioral symptoms were the two common used criteria to diagnose RDSP in all the classification systems in child psychiatry (DC:0-3 [2], DC:0-3R [1] and ICDL-DMIC [11]).

2. To analyze the association between sensory and behavioral symptoms in two clinical groups (RDSP vs. OD3R). It can be assumed that sensory and behavioral symptoms reflect different conditions. It can also be assumed that the associations among these symptoms will be higher in children with RDSP than in those with OD3R.

3. Analysis of the role played by sensory and behavioral symptoms in the diagnosis of RDSP. The hypothesis is that sensory symptoms are just as, if not more, necessary than behavioral symptoms to make RDSP diagnoses.

\section{Method}

Participants

A sequential sample of 161 children between 18 and 36 months of age with diagnoses in Axis I/II of the DC:0-3R; 
Table 1 Diagnoses on the DC:0-3R $(n=78)$ according to category

\begin{tabular}{|c|c|c|c|c|c|}
\hline \multicolumn{3}{|l|}{ Axis I } & \multicolumn{3}{|l|}{ Axis II } \\
\hline Primary diagnosis & $\mathrm{n}$ & $\%$ & Parent-infant relationship global assessment scale & $\mathrm{n}$ & $\%$ \\
\hline Disorders of affect & 14 & 17.9 & 0-10 Documented maltreatment & 1 & 1.3 \\
\hline Adjustment disorders & 11 & 14.1 & 11-20 Grossly impaired & 5 & 6.4 \\
\hline Regulatory disorders & 18 & 23.1 & 21-30 Severely disordered & 13 & 16.7 \\
\hline Sleep behavior disorder & 9 & 11.5 & 31-40 Disordered & 11 & 14.1 \\
\hline Eating behavior disorder & 4 & 5.1 & 41-50 Disturbed & 16 & 20.5 \\
\hline Postraumatic stress disorder & 1 & 1.3 & 51-60 Distressed & 12 & 15.4 \\
\hline Other disorders & 2 & 2.6 & $>60$ (not clinically significant) & 17 & 21.8 \\
\hline Subtotal disorder in Axis I & 59 & 75.6 & Subtotal diagnosis in Axis II & 58 & 74.4 \\
\hline \multirow[t]{2}{*}{ Without disorder in Axis I } & 19 & 24.4 & Without diagnosis in Axis II & 17 & 21.8 \\
\hline & & & Without data in Axis II & 3 & 3.8 \\
\hline Total & 78 & 100 & & 78 & 100 \\
\hline
\end{tabular}

was recruited; these children attended the Infancy and Early Childhood Unit (UPI) of the Department of Child and Adolescent Psychiatry at the Pediatric Hospital in Lisbon between January 2008 and April 2009 as well as between January 2010 and February 2011. Complete data were collected for 148 of these children. Seventy children were excluded due to one or more of the following exclusion criteria: (1) premature birth; (2) previously detected sensory loss (e.g., auditory, visual, among others); (3) parents were not sufficiently proficient in Portuguese to apply the instruments; (4) the presence of specific genetic syndromes; and 5) the presence of Disorders of Relating and Communicating (MSDD) (a similar diagnosis of ASD in DC:0-3R). It is broadly assumed that children with ASD have more severe deficits in general sensory processing than typically developing children [32-35] and those with RDSP [36]. Rates of sensory processing dysfunction may be as high as $90 \%$ in individuals with ASD [32, 37, 38]. They were excluded from this study because there were not enough participants to create a separate group.

A final sample of 78 children (mean age $=29$ months, $53.6 \%$ boys) was obtained. This sample was distributed unevenly among the five socioeconomic levels of the Hollingshead Four Factor Index of Social Status, HFIS [39]; specifically, $2.8 \%$ were from the lower class, $31 \%$ were from the lower-middle class, $12 \%$ were from the middle class, $16.9 \%$ were from the upper-middle class, and $28.2 \%$ were from the upper class. The participants were classified into two groups based on the clinical diagnosis: (described in the Procedure Section). Group 1 ( $\mathrm{n}=18,9$ boys) consisted of children with RDSP, and Group 2 (OD3R) ( $\mathrm{n}=60,32$ boys) consisted of children with a diagnosis in the remaining 5 categories of Axis I (Posttraumatic Stress Disorder, Affect Disorder, Adaptation Disorder, Sleep Disorder and Feeding Disorder) and/or (a relationship-based diagnosis) in Axis II of DC:0-3R (see Table 1).

Procedure

The UPI team members have on average 15 years of clinical experience. This team was composed by three child psychiatrists, a clinical psychologist, two mental health nurses, and an occupational therapist. The UPI team diagnosed children with either RDSP (any Type) or OD3R following the "Decision Tree for diagnosis in Axis I and/or Axis II" of DC:0-3R [1] based on the following information: (1) the data gathered by one of the child psychiatrists during the clinical interview with the parents, (2) the data collected by one of the nurses during the intake interview with the parents, (3) the written report of the parent-child interactions during the clinical interviews and (4) the video recording of the children in a clinical adaptation of the "strange situation" [40] when children were less than 2 years old. The parents who agreed to participate completed the Infant Toddler Sensory Profile, ISTP [36] and Child Behavior Checklist, CBCL 11/2-5 [41] assessment scales, which were independent of the clinical diagnoses. None of the clinicians involved in the diagnosis had access to the results of these assessments. The hospital's ethics committee approved this procedure.

\section{Measures}

\section{CBCL 11/2-5 [41]}

The Portuguese version of the CBCL 1/2-5 [42] was applied. This 100-item, multidimensional, standardized scale assesses children between 18 and 71 months of age. Specifically, this tool measures the frequency with which parents have observed certain behaviors in their children 
over the past 2 months. There are three possible responses on the CBCL 11/2-5: $0=$ "not true", $1=$ "sometimes true", and 2 = "often true". These results are grouped by the following seven syndromes: "emotional reactivity", "anxiety/depression", "somatic complaints", "withdrawn", "sleep problems", "attention problems", and "aggressive behavior". The first four syndromes were grouped as "internalizing problems" category, and the last two symptoms were grouped as "externalizing problems". These scores could also be grouped into five syndromes in the "DSM-oriented scales". In the present study, children with a T-score of 65 or greater on a CBCL $1 \frac{1}{2}-5$ were considered to have displayed significant problematic behaviors (DC:0-3R criteria).

\section{ITSP [36]}

The Portuguese version of the ITSP (Perfil sensorial para bebés e crianças, 7-36 meses [43] was applied. This questionnaire includes 48 items that assess behaviors related to the sensory processing of children who are between 0 and 36 months of age. These items are assessed using a 5-point scale from 1 (almost always) to 5 (hardly ever). This instrument produces results by sensory area and sensory pattern. These results include "low registration", "sensation seeking", "sensory sensitivity", and "sensation avoidance". The sum of the "sensory sensitivity" and "sensation avoidance" pattern scores compose the "low sensory threshold" category. The cut-off scores were determined in the following manner: "Typical performance" corresponded to scores at or between \pm 1 standard deviation (SD) from the mean of children without disabilities, "probable difference" corresponded to scores between $\pm 1 \mathrm{SD}$ and $\pm 2 \mathrm{SD}$ away from the mean, and "definite difference" corresponded to scores \pm 2 SD away from the mean. In the current study, scores greater than 1 SD away from the mean denoted a deficit in sensory modulation (DC:0-3R criteria).

\section{$D C: 0-3 R$ [1]}

This classification is organized into five axes. Axis I consists of the primary diagnosis and the following seven broad diagnostic categories: (1) Traumatic Stress Disorder, (2) Affective Disorders, (3) Adjustment Disorder, (4) Regulation Disorder of Sensory Processing, (5) Sleep Behavior Disorder, (6) Eating Behavior Disorder, (7) MSDD. Each Axis II diagnosis highlights a relational pattern (see Table 1). The severity of these disorders was assessed using the Parent-Infant Relationship Global Assessment Scale (PIR-GAS), the scores of which range from well-adapted (91-100) to documented maltreatment [1]. In the current study, ratings below 60 were considered to be clinically relevant (see Table 1). In addition, Axis III consists of medical and developmental problems. Other supplemental information provided in Axis IV and Axis V was not used in the present study.

\section{HFSI [39]}

This scale categorizes the socioeconomic status (SES) of a family into five classes (lower, low-middle, middle, uppermiddle, and upper) based on each parent's level of education and type of employment.

\section{Clinical Adaptation of "Strange Situation" (UPI Paradigm)}

The original purpose of exposing a child to a strange situation was to identify secure, insecure, or disorganized attachment patterns from observations of the child's behavior during episodes of caregiver-child play, especially during their brief separations and subsequent reunions [40].

Based on some studies [44] the "Strange Situation" [40] was adapted to the clinical context. In the present study, the UPI procedure is video recorded. The behavioral quality, affective tone, and psychological involvement are used in the present study to determine the existence of a relationship diagnosis and its type in Axis II of the DC $0-3 R$ according to PIR-GAS ratings [1].

\section{Data Analyses}

Statistical analyses were performed using SPSS version 15. Data were entered twice. The sociodemographic and clinical characteristics of both diagnostic groups were summarized using descriptive statistics. We compared the symptom severity of both groups to complete the first study objective. Because the RDSP group had only 18 patients and some variables did not meet the criteria of normality, we used the non-parametric Mann-Whitney $U$ test. To avoid the Type I error inflation associated with multiple comparisons, Bonferroni corrections were applied to compare behavioral and sensory symptoms [45]. In a later analysis, the behavioral and sensory symptoms were accounted for, and the discriminative capacity between the two groups was statistically significant. We calculated Spearman's correlations to analyze the associations between sensory and behavioral symptoms to complete the second study objective. We used Fisher's $Z$ test to compare the observed correlations across diagnostic groups.

Logistic regressions were applied to determine the combined effect of behavioral and sensory symptoms on a diagnosis of RDSP to complete the third study objective. The clinical diagnosis was the dependent variable 
$(\mathrm{RDSP}=1, \mathrm{OD} 3 \mathrm{R}=0)$. Sensory and behavioral symptoms were independent variables. In accordance with Peduzzi et al.'s guidelines [46], this logistic regression only included two independent variables given the sample sizes. Thus, 12 analyses were performed in which each model included a sensory symptom and a behavioral symptom. The variable selection was based on the full model that initially included all variables. Due to its ease of application and given that multiple hypothesis testing was needed to complete the proposed objectives we used the Bonferroni correction test to control for Type I errors [45].

\section{Results}

There were no significant between-group differences with regard to sex, age, SES, the developmental problems in Axis III of the DC:0-3R: language delay (LD), or development delays symptoms (DDS), or the presence of diagnosis in Axis II.

\section{Objective 1}

The behavioral characteristics that better distinguished the RDSP group from the OD3R group were the Pervasive Developmental Problems (PDP) (DSM-oriented scale in

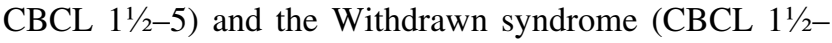
$5)$; the scores for these behavioral characteristics were higher in the RDSP group (see Table 2). When comparing both groups, a tendency towards significance was observed for the symptoms of attention problems and other externalizing problems in CBCL 1 1/2-5.

Almost all sensory symptoms showed a strong capacity to distinguish between groups, and revealed a greater severity in RSDP. These sensory modulation alterations occurred in visual, sensory-oral, and auditory areas as well as in the low registration pattern, sensory avoidance, and low sensory threshold. There was a tendency towards significance in the sensation seeking $(p=0.021)$ and sensory sensitivity patterns $(p=0.026$; see Table 3$)$.

\section{Objective 2}

The PDP and the Withdrawn syndrome in CBCL, correlated higher with most of the sensory symptoms in the RDSP group than in the OD3R group (see Table 4); however, between-group differences in these correlations did not reach significance after applying a Bonferroni correction. The between-group differences whose correlations reached a tendency towards significance $(p$ values $\leq 0.05)$ included the Withdrawn syndrome with the sensory avoidance pattern $(\mathrm{RDSP}=0.67 ; \mathrm{OD} 3 \mathrm{R}=0.15 ; \mathrm{Z}=2.27, p=0.02)$. The same trend occurred between PDP and deficits in auditory processing $(\mathrm{RDSP}=0.44 ; \quad \mathrm{OD} 3 \mathrm{R}=-0.09$;
$\mathrm{Z}=1.94, p=0.05)$. There was also a tendency towards significance between the Attention Deficit/Hyperactivity syndrome and Externalizing problems with regard to alterations in the sensory modulation of the visual area. This correlation occurred in both diagnostic groups but was higher in children with OD3R (0.42) than in those with $\operatorname{RDSP}(\mathrm{r}=-0.16 ; \mathrm{Z}=-2.10, p=0.04)$.

\section{Objective 3}

Regarding the effect of sensory and behavioral symptoms on the diagnosis of RDSP, the results showed that three of the analyzed sensory areas (auditory, visual, and oral) had a significant effect $(p \leq 0.008)$ in the model. Specifically, these areas increased the likelihood of an RDSP diagnosis versus an OD3R diagnosis (see Table 5). The greatest effect was with regard to the visual area $(\mathrm{OR}=4.29, \mathrm{CI}=$ 1.66-11.04), both in the model that included PDP and in the model that included the Withdrawn syndrome $(\mathrm{OR}=4.12$, $\mathrm{CI}=1.59-10.65)$. The effect of the auditory area was similar when the model included either PDP $(\mathrm{OR}=3.10$, $\mathrm{CI}=1.40-6.85)$ or Withdrawn syndrome $(\mathrm{OR}=3.00$, $\mathrm{CI}=1.37-6.56)$. These results are similar to those for the oral area with regard to either the model with PDP $(\mathrm{OR}=3.04, \mathrm{CI}=1.41-6.53)$ or Withdrawn syndrome $(\mathrm{OR}=2.96, \mathrm{CI}=1.38-6.35)$.

The sensory patterns showed a tendency towards significance $(p \leq 0.05)$ and a clinically significant association with regard to the differential diagnoses of RDSP and OD3R. The effect of the sensory avoidance pattern and the low sensory threshold category on the model was positive, indicating a greater likelihood of a diagnosis of RDSP than of OD3R. Moreover, the models with PDP and Withdrawn syndrome reduced the likelihood of an RDSP diagnosis versus an OD3R diagnosis in the case of a low registration behavior pattern (see Table 5). After applying the Bonferroni corrections, it was not found a significant effect of the Withdrawn syndrome and PDP on the diagnosis of RDSP (although several models had $p$ values $\leq 0.05$ ).

The results of this study confirmed most of our hypotheses. Each hypothesis is discussed below

\section{Discussion}

Discriminative Sensory Symptoms of RDSP Versus Those of OD3R

In the present study, the finding that sensory modulation deficits, which were more severe in the RDSP group, were able to make distinctions between the two diagnostic groups was important in terms of the validation of the RDSP diagnosis; therefore, sensory modulation deficits are 
Table 2 Behavioral and emotional symptomatology scores by diagnostic group

\begin{tabular}{|c|c|c|c|c|c|c|c|c|c|}
\hline \multirow[t]{2}{*}{ CBCL $1 \frac{1}{2}-5$} & \multicolumn{4}{|l|}{ RDSP } & \multicolumn{4}{|l|}{ OD3R } & \multirow[t]{2}{*}{$p^{\mathrm{a}}$} \\
\hline & Mean & $\mathrm{SD}$ & $\mathrm{Me}$ & $\left(\mathrm{P}_{25}-\mathrm{P}_{75}\right)$ & Mean & SD & $\mathrm{Me}$ & $\left(\mathrm{P}_{25}-\mathrm{P}_{75}\right)$ & \\
\hline \multicolumn{10}{|l|}{ Syndromes } \\
\hline Emotionally Reactive & 60.89 & 6.69 & 63.5 & $(50-69)$ & 61.83 & 9.09 & 62.0 & $(50-90)$ & 0.867 \\
\hline Anxious/Depressed & 56.72 & 7.38 & 56.0 & $(50-74)$ & 60.30 & 8.73 & 59.0 & $(50-83)$ & 0.102 \\
\hline Somatic Complaints & 58.39 & 7.17 & 58.0 & $(50-74)$ & 57.18 & 7.80 & 53.0 & $(50-80)$ & 0.401 \\
\hline Withdrawn & 64.67 & 11.35 & 63.0 & $(50-85)$ & 56.73 & 7.81 & 56.0 & $(50-79)$ & $0.003^{\mathrm{b}}$ \\
\hline Sleep Problems & 61.17 & 12.11 & 59.0 & $(50-94)$ & 64.92 & 13.31 & 63.0 & $(50-100)$ & 0.250 \\
\hline Attention Problems & 61.06 & 6.51 & 62.0 & $(50-73)$ & 56.98 & 7.49 & 53.0 & $(50-77)$ & 0.017 \\
\hline Aggressive Behavior & 63.61 & 9.42 & 63.0 & $(50-88)$ & 60.87 & 10.22 & 58.0 & $(50-86)$ & 0.149 \\
\hline \multicolumn{10}{|l|}{ (DSM-Oriented Scales) } \\
\hline Affective Problems & 59.72 & 7.71 & 60.0 & $(51-75)$ & 60.88 & 7.39 & 60.0 & $(50-82)$ & 0.538 \\
\hline Anxiety Problems & 60.17 & 9.80 & 60.0 & $(50-84)$ & 63.17 & 9.58 & 63.0 & $(50-84)$ & 0.250 \\
\hline Pervasive Developmental P. & 67.33 & 10.43 & 69.0 & $(51-86)$ & 59.17 & 8.83 & 56.0 & $(50-81)$ & $0.003^{\mathrm{b}}$ \\
\hline Attención Deficit/Hyperactivity P. & 60.78 & 7.72 & 60.0 & $(50-71)$ & 59.00 & 8.36 & 57.0 & $(50-76)$ & 0.390 \\
\hline Oppositional Defiant P. & 62.72 & 8.72 & 64.0 & $(50-80)$ & 59.83 & 9.03 & 57.0 & $(50-80)$ & 0.208 \\
\hline \multicolumn{10}{|l|}{ Problems } \\
\hline Internalizing & 61.56 & 7.46 & 62.0 & $(49-73)$ & 59.28 & 10.15 & 61.0 & $(37-81)$ & 0.521 \\
\hline Externalizing & 63.00 & 8.69 & 63.5 & $(44-83)$ & 59.27 & 10.26 & 56.5 & $(35-85)$ & 0.058 \\
\hline Total & 63.17 & 7.54 & 63.0 & $(45-76)$ & 61.20 & 9.34 & 60.0 & $(43-88)$ & 0.267 \\
\hline
\end{tabular}

${ }^{a}$ Mann-Whitney $\mathrm{U} p$ value

b Significant $p$ values after applying Bonferroni correction test

Table 3 Sensory symptomatology scores by diagnostic group

\begin{tabular}{|c|c|c|c|c|c|c|c|}
\hline & \multicolumn{3}{|c|}{$\operatorname{RDSP}(\mathrm{n}=18)$} & \multicolumn{3}{|c|}{ OD3R $(n=60)$} & \multirow{2}{*}{$p^{\mathrm{a}}$} \\
\hline & Mean Ranks & $\mathrm{Me}$ & $\left(\mathrm{P}_{25}-\mathrm{P}_{75}\right)$ & Mean ranks & $\mathrm{Me}$ & $\left(\mathrm{P}_{25}-\mathrm{P}_{75}\right)$ & \\
\hline \multicolumn{8}{|l|}{ ITSP (sensory areas) } \\
\hline Auditory area & 52.36 & 1.63 & $(0.08-4.19)$ & 35.64 & 0.67 & $(0.02-3.04)$ & $0.006^{\mathrm{b}}$ \\
\hline Visual area & 56.78 & 1.02 & $(0.18-3.14)$ & 34.32 & 0.58 & $(0.01-2.20)$ & $>0.001^{\mathrm{b}}$ \\
\hline Tactile area & 47.42 & 1.17 & $(0.03-2.86)$ & 37.13 & 0.64 & $(0.03-3.82)$ & 0.091 \\
\hline Vestibular area & 47.00 & 1.09 & $(0.10-3.57)$ & 37.25 & 0.71 & $(0.10-3.57)$ & 0.109 \\
\hline Oral area & 55.47 & 2.39 & $(0.78-3.99)$ & 34.71 & 1.33 & $(0.32-3.49)$ & $0.001^{2}$ \\
\hline \multicolumn{8}{|l|}{ ITSP (sensory patterns) } \\
\hline Low registration & 23.14 & 0.53 & $(0.00-1.86)$ & 44.41 & 1.17 & $(0.14-2.25)$ & $>0.001^{\mathrm{b}}$ \\
\hline Sensation seeking & 50.31 & 2.91 & $(0.82-0.40)$ & 36.26 & 1.86 & $(0.16-5.22)$ & 0.021 \\
\hline Sensory sensitivity & 49.94 & 3.02 & $(0.12-5.84)$ & 36.37 & 1.62 & $(0.11-5.79)$ & 0.026 \\
\hline Sensation avoidance & 54.17 & 1.30 & $(0.4-3.41)$ & 35.10 & 0.62 & $(0.00-2.21)$ & $0.002^{\mathrm{b}}$ \\
\hline Low sensory threshold & 54.67 & 1.22 & $(0.12-3.67)$ & 34.95 & 0.62 & $(0.00-3.14)$ & $0.001^{\mathrm{b}}$ \\
\hline
\end{tabular}

${ }^{a}$ Mann-Whitney $\mathrm{U} p$ value

b Significant $p$ values after applying Bonferroni correction test

a differential characteristic of RDSP. These between-group differences were highly distinguishable with regard to three sensory areas: auditory, visual, and oral.

There were two sensory patterns with between-group differences, a high sensory threshold in children characterized one difference, and a low sensory threshold characterized the other. This finding suggests that oscillations in the sensory thresholds of these children are higher compared with other child psychiatric diagnoses. This result is consistent with the first psychophysiological studies conducted using infants and young children with RD symptoms, in which different patterns of physiological 
Table 4 Difference between the RDSP and OD3R groups with regard to correlations between sensory symptoms (with absolute standardized values) and behavioral symptoms in CBCL $1 \frac{1}{2}-5$

\begin{tabular}{lllcccccc}
\hline & & $n$ & Auditory area & Visual area & Oral area & Low registration & Sensation avoidance & Low sensory threshold \\
\hline RDSP & Withdrawn & 18 & 0.39 & 0.47 & 0.36 & -0.44 & 0.67 & 0.57 \\
OD3R & Withdrawn & 60 & -0.02 & 0.23 & 0.06 & -0.34 & 0.15 & 0.21 \\
& & $\mathrm{p}$ & 0.14 & 0.34 & 0.27 & 0.68 & 0.02 & 0.13 \\
RDSP & PDP & 18 & 0.44 & 0.42 & 0.4 & -0.27 & 0.46 & 0.46 \\
OD3R & PDP & 60 & -0.09 & 0.21 & 0.01 & -0.32 & 0.13 & 0.35 \\
& & $\mathrm{p}$ & 0.05 & 0.42 & 0.15 & 0.85 & 0.21 & 0.65 \\
RDSP & Attention Problems & 18 & 0.35 & -0.01 & 0.27 & -0.18 & -0.04 & 0.06 \\
OD3R & Attention Problems & 60 & 0.04 & 0.43 & 0.05 & -0.44 & 0.1 & 0.09 \\
& & $\mathrm{p}$ & 0.26 & 0.11 & 0.43 & 0.32 & 0.63 & 0.60 \\
RDSP & Externalizing P. & 18 & 0.22 & -0.16 & 0.11 & -0.05 & 0.21 & 0.13 \\
OD3R & Externalizing P. & 60 & -0.18 & 0.42 & 0.09 & 0.23 & 0.19 & 0.21 \\
& & $\mathrm{p}$ & 0.16 & 0.04 & 0.94 & 0.33 & 0.94 & 0.78 \\
\hline
\end{tabular}

Table 5 Models with the sensory and behavioral symptom effects on RDSP diagnosis

\begin{tabular}{|c|c|c|c|c|c|}
\hline \multirow[t]{2}{*}{ Model } & \multirow[t]{2}{*}{ Symptoms } & \multirow[t]{2}{*}{ OR } & \multicolumn{2}{|c|}{ OR I.C. $95 \%$} & \multirow[t]{2}{*}{$p$} \\
\hline & & & L. Inf. & L. Sup. & \\
\hline \multirow[t]{2}{*}{1} & Withdrawn syndrome ${ }^{\mathrm{a}}$ & 1.07 & 1.01 & 1.14 & 0.032 \\
\hline & Auditive area & 3.00 & 1.37 & 6.56 & $0.006^{\mathrm{c}}$ \\
\hline \multirow[t]{2}{*}{2} & Withdrawn syndrome & 1.05 & 0.99 & 1.12 & 0.118 \\
\hline & Visual area & 4.12 & 1.59 & 10.65 & $0.003^{\mathrm{c}}$ \\
\hline \multirow[t]{2}{*}{3} & Withdrawn syndrome & 1.06 & 1.00 & 1.13 & 0.056 \\
\hline & Oral area & 2.96 & 1.38 & 6.35 & $0.005^{\mathrm{c}}$ \\
\hline \multirow[t]{2}{*}{4} & Withdrawn syndrome & 1.06 & 1.00 & 1.13 & 0.057 \\
\hline & Low registration pattern & 0.20 & 0.06 & 0.73 & 0.015 \\
\hline \multirow[t]{2}{*}{5} & Withdrawn syndrome & 1.05 & 0.98 & 1.12 & 0.145 \\
\hline & Sensation avoidance pattern & 2.86 & 1.22 & 6.68 & 0.015 \\
\hline \multirow[t]{2}{*}{6} & Withdrawn syndrome & 1.06 & 1.00 & 1.13 & 0.054 \\
\hline & Low sensory threshold & 2.36 & 1.10 & 5.05 & 0.027 \\
\hline \multirow[t]{2}{*}{7} & $\mathrm{PDP}^{\mathrm{b}}$ & 1.07 & 1.01 & 1.14 & 0.026 \\
\hline & Auditive area & 3.10 & 1.40 & 6.85 & $0.005^{\mathrm{c}}$ \\
\hline \multirow[t]{2}{*}{8} & PDP & 1.06 & 1.00 & 1.13 & 0.066 \\
\hline & Visual area & 4.29 & 1.66 & 11.04 & $0.003^{\mathrm{c}}$ \\
\hline \multirow[t]{2}{*}{9} & PDP & 1.07 & 1.00 & 1.13 & 0.044 \\
\hline & Oral area & 3.04 & 1.41 & 6.53 & $0.004^{\mathrm{c}}$ \\
\hline \multirow[t]{2}{*}{10} & PDP & 1.07 & 1.00 & 1.14 & 0.043 \\
\hline & Low registration pattern & 0.19 & 0.05 & 0.70 & 0.012 \\
\hline \multirow[t]{2}{*}{11} & PDP & 1.05 & 0.99 & 1.13 & 0.111 \\
\hline & Sensation avoidance pattern & 2.87 & 1.25 & 6.60 & 0.013 \\
\hline \multirow[t]{2}{*}{12} & PDP & 1.06 & 0.99 & 1.13 & 0.076 \\
\hline & Low sensory threshold & 2.31 & 1.05 & 5.07 & 0.037 \\
\hline
\end{tabular}

\footnotetext{
a Withdrawn syndrome in CBCL $1 \frac{1 / 2-5}{2}$

b DSM-oriented scale Pervasive Developmental Problems in CBCL 1 1/2-5

${ }^{\mathrm{c}}$ Significant $p$ values after applying Bonferroni correction test
} 
reactivity were observed in relation to normally developing children $[22,47]$. The results of this study also agree with those of psychophysiological studies that have compared children who have sensory modulation dysfunction (SMD) with normally developing children, with findings such as a higher frequency and amplitude in skin conductance [15], different event-related potential patterns [14], and increased parasympathetic responses [16] with regard to task performance in children with SMD.

\section{Distinct Behavioral RDSP Symptoms}

The most discriminative behavioral symptoms of the RDSP diagnosis with regard to OD3R were Withdrawn syndrome and PDP (DSM-oriented scale in CBCL $1 \frac{1}{2}-5$ ). These two scales in CBCL $1 \frac{1}{2}-5$ were also the more distinctive in another study between children with ASD and children with a typical development [48]. This result brings to mind studies that have reported phenotypic similarities between RDSP and ASD in early childhood [8, 13].

Even though children with ASD were excluded from this study, an explanation for these results can be that some of the symptoms assessed by CBCL like: "Afraid to try new things, disturbed by change, strange behavior, upset by new situations, withdrawn, the children doesn't get along with peers" which are included in the Pervasive Developmental Problems category, are also characteristics of an RDSP diagnostic. Because some similar symptoms exist between RDSP and ASD a careful assessment is fundamental in order to make a differential diagnosis and prevent misdiagnosis of these children.

However, it is important to note that the severity of PDP and Withdrawn syndrome scores in CBCL $1 \frac{1}{2}-5$ were subclinical for both groups in the present study. Other studies that have addressed the differential diagnoses of RDSP and MSDD have shown significant higher scores in CBCL in Withdrawn syndrome and Somatic problems sub-scales in MSDD. In those studies, only the MSDD group reached the clinical cutoff score in Total problems, Internalizing problems and Withdrawn syndrome. Those studies concluded that these diagnoses were separate clinical entities and it supported the idea that these dimensions may facilitate the differential diagnosis of these disorders [13, 49].

In the present study, other symptoms included in the RDSP diagnostic criteria in DC: $0-3 \mathrm{R}$ show a tendency towards significance only in their discriminative ability (e.g., Attention Deficit/Hyperactivity syndrome and other externalizing problems), which suggest that these symptoms are present at similar severities in other early childhood psychiatric diagnoses. This supposition would explain the phenotypic similarities to other conditions reported by previous studies, such as Attention Deficit/ Hyperactivity Disorder [20] and Conduct Disorder [19].
The Relationship Between Sensory and Behavioral Symptoms

The absence of a high correlation between sensory and behavioral symptoms in both groups suggests that these symptoms reflect different conditions, which confirms our hypothesis and is supported by other studies $[25,26,50]$. It is important to note that the correlation between Withdrawn syndrome (on CBCL) and avoidance sensory symptoms (assessed with the ITSP) in children with RDSP was only moderate.

On the other hand, the lack of significant between-group differences does not support the hypothesis that there is a stronger relationship between behavioral and sensory symptoms in children with RDSP versus those with OD3R. However, some correlations showed a tendency towards significance such as that between PDP symptoms and auditory modulation alterations in the RDSP group as well as between the Withdrawn syndrome and sensory avoidance symptoms, which were moderately correlated in the RDSP group (0.67) but weakly correlated in the OD3R group (0.15). The small sample size might explain why this difference was not significant. The only correlation that was stronger in the OD3R group than in the RDSP group was externalizing disorders with alterations in visual processing. As other studies have already suggested [19], deficits in sensory modulation might also be present in children with diagnoses other than RDSP, but to a lesser extent.

The Effect of Sensory and Behavioral Symptoms on an RDSP Diagnosis

The effect of the sensory variables on the RDSP diagnosis was much more significant than that of the behavioral variables. Specifically, the effect of sensory symptoms was significant in the model with the Withdrawn syndrome and in the model with PDP. Conversely, we did not find a significant effect of the behavioral symptoms on RDSP diagnoses. Nevertheless, it is important to note that the present study is not an epidemiological study [25] or a comparison group with children from the general population but a clinical comparison group. Children without behavioral symptoms are not brought to clinical units for treatment.

For that reason, the lack of a behavioral symptoms effect in the model does not mean that these behavioral symptoms are not relevant for making a diagnosis; however, differences in sensory modulation levels primarily allow differential diagnoses in clinical samples. This result found support in the OT classification system which primarily makes the diagnosis of SMD based on sensory criteria [50, 51]. It could be important to consider this preliminary 
result, in the actual classification in OT field and Child Psychiatry field in order to analyze the discriminative ability of behavioral symptoms in other clinical samples.

\section{Limitations}

The instruments used to collect the child information by the researcher were based in the parental perceptions of the behavioral and sensory processing of their children. To a large extent, the adequacy of these perceptions influenced the results found.

The DC:0-3 system needs to be more directly reviewed, particularly with regard to its reliability and validity.

There are also limitations regarding the sample size of the study and the inclusion of children without diagnosis in Axis I but only in Axis II in the sample; therefore, the results must be considered with caution. We interpreted the results conservatively: in all cases, the Bonferroni correction was applied to correct for inflated statistical significance. We cannot state that some of the variables in Objectives 1 and 3 that only showed a tendency towards significance would become statistically significant and result in more definitive differential diagnoses between RDSP and OD3R in a study with a larger sample. The same is true for the correlations between the sensory and behavioral symptoms in Objective 2, in which the small sample size of children with RDSP might have prevented identifying significant between-group differences.

Future studies with a larger sample size would allow researchers to analyze the full logistic regression model to study the predictive and discriminative ability of the respective model with regard to differential RDSP diagnoses.

It would be interesting if future studies were conducted to analyze the stability of RDSP over time and with phenotypic similarities as diagnosed through DSM in preschoolers (i.e. The RDSP Type C-Sensory StimulationSeeking/Impulsive, may be associated with AttentionDeficit Hyperactivity Disorder (DSM-IV-TR), particularly the hyperactive/impulsive type or combined type).

It would also be interesting to study whether it would be possible to unify diagnostic criteria for SMD and RDSP (including both sensory modulation deficits and behavioral criteria) when motor symptoms were present (such as dyspraxia or postural disorders) establishing the possibility to diagnose SBMD like in OT and ICDL-DMIC systems.

\section{Summary}

Regarding the first objective, the results showed a greater severity of sensory modulation deficits in various sensory categories and an increased severity of certain behavioral symptoms in the RDSP group. These findings indicate the presence of the differential characteristics of other early childhood psychiatric disorders. The associations between sensory and behavioral symptoms were low to moderate in children with RDSP and in those with OD3R. In addition, there were no significant between-group differences, suggesting that the RDSP diagnostic criteria are independent.

Regarding the third objective, the results showed that sensory modulation deficits had a greater effect on the RDSP group than on the OD3R group. The results also showed that the sensory variables had a greater effect on the RDSP diagnosis than did the behavioral variables in both child psychiatric samples. The results of this study revealed convergent validity between the instruments and the RDSP diagnostic criteria. Furthermore, they support the validity of RDSP as a single diagnosis in early childhood.

Moreover, these results confirm that there are specific symptoms that should alert practitioners to a possible diagnosis of RDSP. At a clinical level, they support the importance of applying specific instruments for diagnosing RDSP. Obtaining the sensory profile of young children might be especially helpful when it is necessary to confirm a diagnosis if RDSP is suspected in early childhood or to make a differential diagnosis regarding another psychiatric condition. Through early diagnosis, this procedure would benefit young children in need of specific sensory integration techniques and would prevent future psychiatric conditions.

Acknowledgments This research was supported in part by grant SFRH/BD/38992/2007 from the Portuguese Foundation for Science and Technology of the Ministry of Science, Technology and Higher Education, POCI 2010 and an European Social Grant. The authors gratefully acknowledge the clinical team of UPI of CHCL-Hospital Dona Estefânia for their participation and implementation of the research protocol, Marta Alves MSc, from the Epidemiology and Statistics Office of the Research Unit of CHCL, and the parents of the child who participate in this research.

\section{References}

1. Zero to Three (2005) Diagnostic classification: 0-3R: Diagnostic classification of mental health and developmental disorders of infancy and early childhood: revised edition. Zero to Three Press, Washington, DC

2. Zero to Three (1994) Diagnostic classification: 0-3: Diagnostic classification of mental health and developmental disorders of infancy and early childhood. National Center for Clinical Infant Programs, Arlington

3. American Psychiatric Association (2000) Diagnostic and statistical manual of mental disorders, 4th edn. American Psychiatric Association, Washington, DC

4. Rescorla L (2005) Assessment of young children using the Achenbach system of empirically based assessment (ASEBA). Ment Retard Dev D R 11:226-237

5. Reebye P, Stalker A (2007) Regulation disorders of sensory processing in infants and young children. BCMJ 49:194-200 
6. Williamsom GG, Anzalone ME (2001) Sensory integration and self-regulation in infants and toddlers: helping very young children interact with their environment. Zero to three, National Center for Infants Toddlers and their families, Washington, DC

7. DeGangi GA, Porges SW, Sickel RZ, Greenspan SI (1993) Fouryear follow-up of a sample of regulatory disordered infants. Inf Mental Health J 4:330-343

8. DeGangi GA, Breinbauer C, Porges SW, Greenspan SI (2000) Prediction of childhood problems at three years in children experiencing disorders of regulation during infancy. Inf Mental Health J 21:158-175

9. Skovgaard AM, Houmann T, Christiansen E, Landorph S, Jorgensen T, Olsen EM (2007) The prevalence of mental health problems in children $11 / 2$ years of age-the Copenhagen Child Cohort 2000. J Child Psychol Psyc 48:62-70

10. Miller LJ, Robinson J, Moulton D, Sensory Modulation Dysfunction: Identification in Early Childhood (2004) In: DelCarmen-Wiggins R, Carter A (eds) Handbook of infant, toddler, and preschool mental health assessment, pp 247-270

11. Interdisciplinary Council on Developmental and Learning Disorders (2005) Diagnostic manual for infancy and early childhood: mental health, developmental, regulatory-sensory processing, and language disorders and learning challenges (ICDL-DMIC). Author, Bethesda

12. World Health Organization (1994) International and statistical classification of diseases and related health problems (ICD-10, 10th edn. WHO, Geneva

13. Cesari A, Maestro S, Cavallero C, Chilosi A, Peccini P, Pfanner L, Muratori F (2003) Diagnostic boundaries between regulatory and multisystem developmental disorders: a clinical study. Inf Mental Health J 4:365-377

14. Davies PL, Gavin JW (2007) Validating the diagnosis of sensory processing disorders using EEG technology. Am J Occup Ther 61:176-189

15. McIntosh DN, Miller LJ, Shyu V, Hagerman RJ (1999) Sensory modulation disruption, electrodermal responses, and functional behaviors. Dev Med Child Neurol 41:608-615

16. Schaaf RC, Miller LJ, Seawell D, O'Keefe S (2003) Children with disturbances in sensory processing: a pilot study examining the role of the parasympathetic nervous system. Am J Occup Ther 4:442-449

17. Ben-Sasson A, Cermak SA, Orsmond A, Carter AS, Fogg L (2007) Can we differentiate sensory over responsivity from anxiety symptoms in toddlers? Perspectives of occupational therapists and psychologists. Inf Mental Health J 28:536-558

18. Briggs-Gowan MJ, Carter AS, Bosson-Heenan J, Guyer AE, Horwitz SM (2006) Are infant-toddler social-emotional and behavioral problems transient? J Am Acad Child Psy 7:849-858

19. Cheng M, Boggett-Carsjens J (2005) Consider sensory processing disorders in the explosive child: case report and review. Can Child Adolesc Psychiatr Rev 14:44-48

20. Mangeot SD, Miller LJ, McIntosh DN, McGrath-Clarke J, Hagerman RJ, Goldson E (2001) Sensory modulation dysfunction in children with attention deficit hyperactivity disorder. Dev Med Child Neurol 43:399-406

21. James K, Miller LJ, Schaaf R, Nielsen DM, Schoen SA (2011) Phenotypes within sensory modulation dysfunction. Comprehensive Phsychiatry 52:715-724

22. DeGangi GA, DiPietro JA, Greenspan SI, Porges WP (1991) Psychophysiologycal characteristics of the regulatory disordered infant. Infant Behav Dev 37-50

23. Pérez Robles R, Jané Ballabriga MC, Doval Dieguez E, Caldeira da Silva P (2011) Validating regulatory sensory processing disorders in early childhood using the sensory profile and child behaviour checklist (CBCL 1 1/2-5). J Child Fam Stud. doi: 10.1007/s10826-011-9550-4
24. Gunn TE, Tavegia BD, Houskamp BM, McDonald LB, Bustrum JM, Welsh RK (2009) Relationship between sensory deficits and externalizing behaviors in an urban, Latino preschool population. JCFS 18:653-661

25. Gouze KR, Hopkins J, LeBailly SA, Lavigne JV (2009) Reexamining the epidemiology of sensory regulation dysfunction and comorbid psychopathology. J Abnorm Child Psychol 37:1077-1087

26. Van Hulle CA, Schmidt NL, Goldsmith HH (2012) Is sensory over-responsivity distinguishable from childhood behavior problems? A phenotypic and genetic analysis. J Child Psychol Psyc 53:64-72

27. Goldsmith HH, Van Hulle CA, Arnerson CL, Schreiber JE, Gernsbacher MA (2006) A population-based twin study of parentally reported tactile and auditory defensiveness in young children. J Abnorm Child Psychol 34:393-407

28. Kagan J, Snidman N (1991) Infant predictors of inhibited and uninhibited profiles. Psychol Sci 1:40-44

29. Kagan J, Snidman N, Hendler J, Greene SM (1991) Predicting inhibited and uninhibited behaviour. Irish J Psychol 2:248-262

30. Bar-Shalita T, Vatine JJ, Seltzer Z, Parush S (2009) Psychophysical correlates in children with sensory modulation disorder (SMD). Physiol Behav 98:631-639

31. Ognibene TC (2003) Distinguishing sensory modulation dysfunction from Attention-Deficit/Hyperactivity Disorder: Sensory habituation and response inhibition processes. Dissertation Abstracts International: Section B: The Sciences and Engineering 12:6101

32. Tomchek SD, Dunn W (2007) Sensory processing in children with and without autism: a comparative study using the short sensory profile. Am J Occup Ther 61:190-200

33. Ben-Sasson A, Cermak SA, Orsmond GI, Carter AS, Kadlec MB, Dunn W (2007) Extreme sensory modulation behaviors in toddlers with autism. Am J Occup Ther 61:584-592

34. Ben-Sasson A, Cermak SA, Orsmond GI, Tager-Flusberg H, Carter AS, Kadlec MB (2008) Sensory subgroups of toddlers with autism spectrum disorders: differences in internalizing symptoms. J Child Psychol Psyc 49:817-825

35. Baranek GT, David FJ, Poe MD, Stone WL, Watson LR (2006) Sensory experiences questionnaire: discriminating sensory features in young children with autism, developmental delays, and typical development. J Child Psychol Psyc 47:591-601

36. Dunn W (2002) The infant/toddler sensory profile manual. The Psychological Corporation, San Antonio

37. Leekam S, Tandos J, McConachie H, Meins E, Parkinson K, Wright C, Turner M, Arnott B, Vittorini L, Couteur AL (2007) Repetitive behaviours in typically developing 2-year-olds. J Child Psychol Psyc 48:1131-1138

38. Baker A, Lane A, Angley M, Young R (2008) The relationship between sensory processing patterns and behavioural responsiveness in autistic disorder: a pilot study. J Autism Dev Disord 38:867-875

39. Hollingshead AB (1975) Four factor index of social status. Department of Sociology, Yale University, New Haven

40. Ainsworth M (1985) Patterns of infant-mother attachments: antecedents and effects on development. B New York Acad Med 61:771-791

41. Achenbach TM, Rescorla L (2000) Manual for the ASEBA preschool forms \& profiles. Department of Psychiatry, University of Vermont, Burlington

42. Gonçalves M, Dias P, Machado BC (2007) Questionário de Comportamentos da Criança $1 \frac{1 / 2}{2}-5$. Not published manuscript, Universidade do Minho, Braga

43. Pérez Robles R (2008) Perfil sensorial para bebés e crianças (7-36 meses). Not published manuscript, Universidad Autónoma de Barcelona, Barcelona 
44. Lewis M, Feiring C (1989) Infant, mother, and mother-infant interaction behavior and subsequent attachment. Child Dev 60:831-837

45. Bender R, Lange S (2001) Adjusting for multiple testing-when and how? J Clin Epidemiol 54:343-349

46. Peduzzi P, Concato J, Kemper E, Holford TR, Feinstein AR (1996) A simulation study of the number of events per variable in logistic regression analysis. J Clin Epidemiol 49:1373-1379

47. Porges SW (1991) Vagal tone: an autonomic mediator of affect. In: Garber JA, Dodge KA (eds) The development of affect regulation and dysregulation. Cambridge University Press, New York, pp 111-128

48. Sikora DM, Hall TA, Hartley SL, Gerrard-Morris AE, Cagle S (2008) Does parent report of behavior differ across ADOS-G classifications: analysis of scores from the CBCL and GARS. J Autism Dev Disord 38:440-448

49. Aron EN, Aron A (1997) Sensory-processing sensitivity and its relation to introversion and emotionality. J Pers Soc Psychol 73:345-368

50. Dunn W (2001) The sensations of everyday life: empirical, theoretical, and pragmatical considerations, Eleanor Clarke Slagle lecture. Am J Occup Ther 55:608-620

51. Miller LJ, Anzalone ME, Lane SJ, Cermak SA, Osten ET (2007) Concept evolution in sensory integration: a proposed nosology for diagnosis. Am J Occup Ther 61:135-140 